\title{
TITLE:
}

\section{A Dynamical Theory of Cascade in Turbulence and Non-Gaussian Statistics}

$\operatorname{AUTHOR}(S)$ :

KAMBE, T.

\section{CITATION:}

KAMBE, T.. A Dynamical Theory of Cascade in Turbulence and Non-Gaussian Statistics. 数 理解析研究所講究録 1991, 769: 156-171

ISSUE DATE:

1991-11

URL:

http://hdl.handle.net/2433/82342

RIGHT: 
数理解析研究所講究録

第 769 巻 1991 年 $156-171$

156

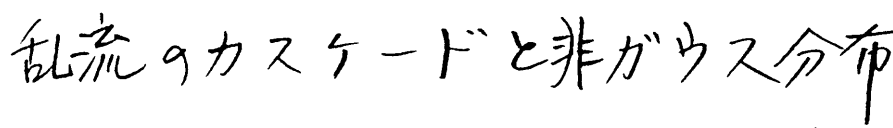

(要部)

東大理神部免

よく知られている乱流のカスケードと速度こう配の非がウ 又分布統計とを結びつけるーつの父イナミカルなモデル機構

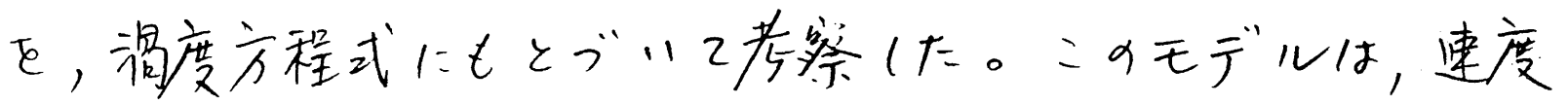
場を解析的な成分と, 非整数のス个一儿指数をもつ特異的成

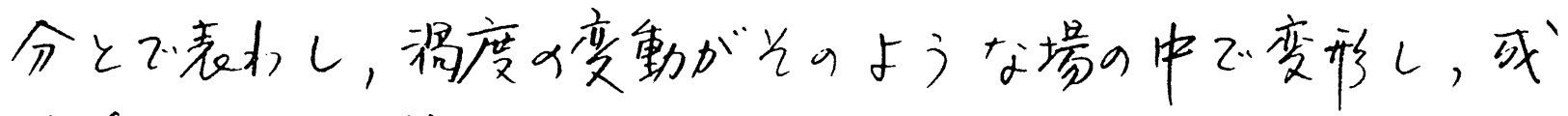

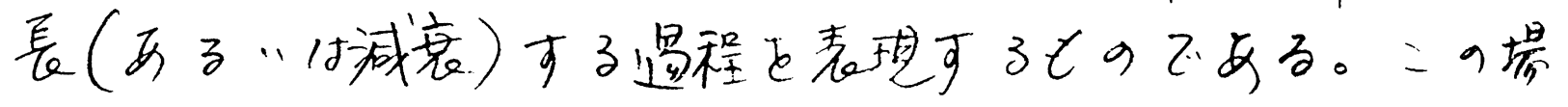

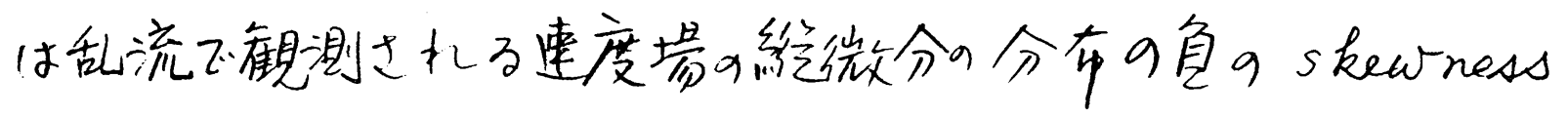
の性筧をもたせることがでする。

上小よう場で、滑度变動のカスケ一ドを記述する非淥形壬 デル方程式が導かれた。本モデルは横微分・维微分の統部的 性筧9相㝵を導きささら微分の倩数と共にflatress factor が增加する性篦を示可。数檤シシュレーンョンと比較( る, 大きな予循は县い出されていない。 


\section{A Dynamical Theory of Cascade in Turbulence and Non-Gaussian Statistics}

\section{T. KAMBE}

Department of Physics, University of Tokyo, Bunkyo-ku, Tokyo 113

\section{Introduction}

A dynamical mechanism is considered which connects the cascade with nongaussian statistics of velocity gradients. Turbulence is characterized by the continuous excitation of all scales, but in the Fourier space of the velocity field, the excited amplitude decreases rapidly with increasing wave numbers so that contribution to the total kinetic energy from the small scale components is negligibly small. Roughly speaking by the central limit theorem, the sum of a large number of Fourier modes is distributed normally when the Fourier amplitudes of different wave numbers are independent in the energy-containing eddies (Batchelor 1953).

However it is well-known that non-gaussian statistics are observed at small scales. Two simplest measures of non-gaussianity are the skewness and flatness.

(a) Experimental observations concerning the small-scale motion show that the flatness of the probability distribution of the various velocity derivatives increases steeply with the order of the derivative. For example, the flatness factor of the $n$-th order longitudinal derivative $g_{n}=\partial^{n} u / \partial x^{n}$ for a coordinate $x$ and the corresponding velocity $u$, defined as $F_{n}=\bar{g}_{n}^{4} /\left(\bar{g}_{n}^{2}\right)^{2}$, shows the value of about $3.9,4.9$ and 5.9 (for $n=1$, 2 and 3 respectively: Batchelor 1953; Monin \& Yaglom 1975) which are greater than the gaussian value 3 , where the over-bar denotes the ensemble average. A large flatness factor of a distribution implies that the probability density function has a higher central peak and broader skirts than the gaussian function of the same standard deviation. These properties are considered to be intrinsic to the micro-structures of turbulence in general.

(b) The measurements of the skewness factor $\overline{g_{1}^{3}} /\left(\overline{g_{1}^{2}}\right)^{3 / 2}$ suggest that the value is around $-0.3 \sim-0.5$. The probability density of $g_{1}$ is characterized by the properties that small positive values of $g_{1}^{3}$ are more probable than small negative values, but this positive contribution to $\overline{g_{1}^{3}}$ is less than balanced by the larger contribution of negative large values of $g_{1}^{3}$ as compared with positive ones.

In the study of numerical simulation of incompressible Navier-Stokes turbulence at moderately large Reynolds number, She et al. (1988) isolated the contributions 
of different flow scales by considering Fourier-band filtering of the velocity field, and investigated the non-gaussian behaviors. In particular the probability distribution functions are found to have near-exponential forms for the velocity and its derivatives. Exponential behaviors of the distribution functions are observed in various context of experiments and numerical simulations (e.g. Van Atta \& Chen 1970; Sheih et al. 1971; Yamamoto \& Hosokawa 1988; Kida \& Murakami 1989; Gagne 1990). These properties are examined also by the statistical analysis (Yamamoto \& Kambe 1991) about the contributions from different Fourier subspaces of a decaying turbulence obtained numerically.

Kraichnan (1990) considered a heuristic model for evolution of the probability distribution of velocity gradient and an exponential distribution. The idea is that an initial gaussian field is mapped dynamically into a non-gaussian field. The initial statistics of the fluctuations are preserved during the dynamical cascade. The dynamics is governed by a nonlinear ordinary differential equation that models competition between the inertial straining and viscous damping and produces small scales. Similar nonlinear evolution equation has been considered by She (1991). His model equation is distilled from the vorticity equation by taking account of a random background field and a dynamical exponent $\alpha$ for a local self-stretching mechanism with a scaling argument.

Study of the vorticity dynamics based on the vorticity equation for a viscous incompressible fluid was performed previously for arbitrary flat shear layers (Kambe 1983,1986 ) or an arbitrary axisymmetric shear layer (Kambe 1984). In these analyses, motions of rotational (shear) layers superimposed on irrotational straining field are investigated, in which the dynamical evolution of the vorticity proceeds under the influence of three elemental processes: convection, stretching and viscous diffusion. With using local expressions of the background straining velocity field which are linear with respect to the space coordinates, it is found to be able to represent the evolution of the rotational layers exactly in terms of the initial vorticity distribution. This formulation can describe exponential growth of the vorticity when the viscous term is neglected. However this is contrasted with the algebraically explosive behaviors which are known in the solution of the quadratically nonlinear model equation of the vorticity (Rose \& Sulem 1978) or the equation for the enstrophy in the statistical theory of turbulence with the quasi-normal approximation (Proudman \& Reid 1954). The present study is an endeavor to improve the previous study by incorporating the property of scale invariance of turbulence field described below. ${ }^{1}$

\footnotetext{
${ }^{1}$ The idea has been inspired by the presentation of She $(1991 \mathrm{a}, \mathrm{b})$ at the international workshop held in Japan, October 1990. His model equation includes the effect of scale invariance.
} 
We investigate a dynamical mechanism that connects the cascade in turbulence with the near-exponential tail in the distribution of velocity and their derivative fields. It will be shown below that the difference in behavior between the lateral and longitudinal derivatives may lead to different probability distributions: slower-than or steeper-than exponential decay.

One of the remarkable symmetries of the Euler equation is the scale invariance which is considered by Frisch \& Parisi (1985) in relation to a multifractal model of turbulence that they introduced. This property is as follows. The Euler equation is invariant if we simultaneously scale the distance by $\lambda$, the velocity by $\lambda^{h}$ and the time by $\lambda^{1-h}$ (the pressure by $\lambda^{2 h}$ ), where $h$ is an arbitrary local scaling exponent. Recent wavelet analysis of an experimental data suggests $-0.5<h<1.0$ (Bacry et al. 1990). The Kolmogorov's self-similar cascade corresponds to the value $h=\frac{1}{3}$ which describes scale invariance of the rate of energy transfer between eddies of different scales.

\section{Straining of a test field by large-scale fields}

\subsection{Local representation}

Taking account of the features of turbulence described in the introduction, we try to construct a model of cascade in turbulence. We consider the vorticity equation,

$$
\omega_{t}+(\mathbf{v} \cdot \nabla) \omega-(\omega \cdot \nabla) \mathbf{v}=\nu \nabla^{2} \omega
$$

where $\mathbf{v}$ is the velocity and $\boldsymbol{\omega}=\nabla \times \mathbf{v}$ is the vorticity. Suppose that the velocity field $(u, v, w)$ is locally represented in the cartesian coordinate system $(x, y, z)$ as

$$
\begin{aligned}
u & =A x-\Psi_{z}(x, z)-\psi_{y}(x, y, t) \\
v & =B y+\psi_{x}(x, y, t) \\
w & =C z+\Psi_{x}(x, z)
\end{aligned}
$$

near the origin $(0,0,0)$, where $A, B, C$ are constants with the constraint relation $A+B+C=0$. The function $\Psi(x, z)$ satisfies the equation, $\Psi_{x x}+\Psi_{z z}=0$, hence $\Psi(x, z)$ being a streamfunction describing a steady incompressible irrotational flow in $x, z$ plane, while $\psi(x, y, t)$ is a time-dependent streamfunction describing a rotational flow in $x, y$ plane, hence $\psi_{x x}+\psi_{y y} \neq 0$, and considered to be a rotational perturbation to the steady irrotational field $\Psi(x, z)$. Obviously the velocity field (2) satisfies the solenoidal condition, $\operatorname{div} \mathbf{v}=0$. The velocity consists of three components: a regular irrotational field $\mathbf{v}^{(r)}=(A x, B y, C z)$, a singular irrotational field $\mathbf{v}^{(s)}=\left(-\Psi_{z}, 0, \Psi_{x}\right)$ 
whose functional form is given in the next subsection, and an unsteady test field $\mathrm{v}^{(t)}=\left(-\psi_{y}, \psi_{x}, 0\right)$. It is readily shown that the vorticity has only the $z$ component associated with $\psi$ :

$$
\boldsymbol{\omega}=(0,0, \omega), \quad \omega(x, y, t)=\psi_{x x}+\psi_{y y} \quad .
$$

We consider the evolution of the test field $\psi(x, y, t)$ strained by the background singular field $\mathbf{v}^{(s)}$ and the regular field $\mathbf{v}^{(r)}$, which are supposed to be of the scale of inertial range and larger one, respectively. It is important that the singular component $\mathbf{v}^{(s)}$ is introduced here to represent the turbulent field having the scale invariance mentioned in the end of the previous section. In turbulence, the field $\mathbf{v}^{(s)}$ would be quasi-steady in comparison with the rapid cascading process of fluctuations.

The $x$ and $y$ components of the vorticity equation (1) vanish identically. Using the explicit expression of the velocity (2), we have

$$
\frac{\partial \omega}{\partial t}+A x \frac{\partial \omega}{\partial x}+B y \frac{\partial \omega}{\partial y}-C \omega-\frac{\partial}{\partial x}\left(\Psi_{z} \omega\right)+\frac{\partial(\psi, \omega)}{\partial(x, y)}=\nu \nabla^{2} \omega
$$

for the $z$ component $\omega=\psi_{x x}+\psi_{y y}$.

\subsection{Singular irrotational field}

Based on the view that straining of the test field by only the regular component $v^{(r)}$ is insufficient to describe the cascade in turbulence, we introduce a singular component $\mathbf{v}^{(s)}$ having the scale-invariant property. Assume that

$$
\Psi(x, z)=\sum_{n=0}^{\infty} a_{n} x^{h-2 n} z^{2 n+1}=a x^{h} z\left(1+\zeta_{1} \frac{z^{2}}{x^{2}}+\zeta_{2} \frac{z^{4}}{x^{4}}+\cdots\right)
$$

where $h$ is a fractional parameter in the range $-0.5<h<1.0, a=a_{0}$ and $\zeta_{n}=a_{n} / a_{0}$. The irrotationality condition $\Psi_{x x}+\Psi_{z z}=0$ leads to

$$
\zeta_{n} \equiv \frac{a_{n}}{a_{0}}=(-1)^{n} \frac{\{2 n-1-h\}}{(2 n+1) !},
$$

where $\{n-h\} \equiv(n-h)(n-1-h) \cdots(1-h)(-h)$ for an integer $n$. This series solution converges for $|z / x|<1$, but has a singular behavior at $x=0$ due to the terms of the form $x^{h-2 n}$ with a fractional power $h-2 n$. Here we restrict our consideration to the part of positive $x$ : $0<|z|<x$. Clearly the solution (4) satisfies the viscous vorticity equation identically in the region of definition because of the vanishing vorticity.

The velocity fields, i.e. $x$ and $z$ components, represented by the streamfunction $\Psi(x, z)$ are

$$
-\Psi_{z}=-a x^{h}\left(1+3 \zeta_{1} \frac{z^{2}}{x^{2}}+5 \zeta_{2} \frac{z^{4}}{x^{4}}+\cdots\right)
$$




$$
\Psi_{x}=a x^{h-1} z\left(h+(h-2) \zeta_{1} \frac{z^{2}}{x^{2}}+(h-4) \zeta_{2} \frac{z^{4}}{x^{4}}+\cdots\right)
$$

respectively. It is evident that the above velocities are scaled by $\lambda^{h}$ for the simultaneous scaling of $x$ and $z$ by $\lambda$.

\subsection{Test field $\psi(x, y, t)$}

Consider a test field whose vorticity $\omega$ is localized and characterized by a length scale $\lambda(t)$. The scale $\lambda(t)$ is supposed to decrease by the straining of the background fields. First we assume that the fluctuation $\psi(x, y, t)$ is represented in the form

$$
\psi(x, y, t)=F(x, t)
$$

for simplicity. (Later we will consider the case, $\psi(x, y, t)=y F(x, t)$ which shows an additional property of the test field). Further we assume that the vorticity $\omega(x, t)=$ $F_{x x}$ is given by the form,

$$
F_{x x}(x, t)=s_{0}\left(\frac{\lambda_{0}}{\lambda(t)}\right)^{\gamma} f(\xi)
$$

where

$$
\xi=\frac{x-\lambda \xi_{0}}{\lambda}, \quad \text { or } \quad x=\lambda\left(\xi+\xi_{0}\right),
$$

$\xi_{0}$ being a constant. The coefficient $s_{0}$ denotes a typical initial value of the test field vorticity. Note that the assumption (6) is given a special form to describe the cascade process in which the scale parameter $\lambda(t)$ is supposed to decrease. This is, in a sense, a phenomenological setup on which the subsequent consideration is based.

\subsection{Velocity fields}

Now the velocity components are given by

$$
u=A x-\Psi_{z}, \quad v=B y+F_{x}, \quad w=C z+\Psi_{x}
$$

where $\Psi$ is approximated by $a x^{h} z$ with a positive parameter $a$. From this we obtain the longitudinal derivatives,

$$
\frac{\partial u}{\partial x}=A-\Psi_{x z}, \quad \frac{\partial v}{\partial y}=B, \quad \frac{\partial w}{\partial z}=C+\Psi_{x z}
$$

where $\Psi_{x z}=a h x^{h-1}$. The lateral derivatives are

$$
\frac{\partial u}{\partial z}=-\Psi_{z z} \sim-x^{h-2} z, \quad \frac{\partial v}{\partial x}=F_{x x}, \quad \frac{\partial w}{\partial x}=\Psi_{x x} \sim x^{h-2} z .
$$


The other lateral derivatives vanish. Since the vorticity has only the $z$ component, the rate of vortex stretching is given by the longitudinal derivative $\partial w / \partial z=C+\Psi_{x z}$.

Consider a favorable case for the vortex stretching, that is $C>0$ and $A, B<0$. Cubic sum of the longitudinal derivatives is

$$
S_{3}=\left(A-\Psi_{x z}\right)^{3}+B^{3}+\left(C+\Psi_{x z}\right)^{3}=3 A B C+3\left(C^{2}-A^{2}\right) \Psi_{x z}-3 B \Psi_{x z}^{2}
$$

where $A+B+C=0$ is used. Although each derivative of (9) takes both negative or positive value, the sum $S_{3}$ of the three cubic terms is found to be positive since each term on the right hand side is positive due to the assumed properties. Consideration of the relation to the turbulence statistics is given in the section 3.2 .

Let us consider a fluid particle advected passively by the flow, whose motion is denoted as $(X(t), Y(t), Z(t))$. The $x, y$ positions must satisfy the equation,

$$
\dot{X}=A X-\Psi_{z}(X, Z), \quad \dot{Y}=B Y+F_{x}(X, t),
$$

where the over dot means the time derivative with respect to the fixed particle in motion and $\Psi_{z}$ is approximated by $a x^{h}$. A small $x$-separation $\Delta_{X}(t)=X_{1}(t)-X_{2}(t)$ between two nearby particles marked as 1 and 2 will be governed by

$$
\dot{\Delta}_{X}=\left(A-\Psi_{x z}(\bar{X}, Z)\right) \Delta_{X}
$$

where $\bar{X}=\left(X_{1}+X_{2}\right) / 2$.

\subsection{A model of cascade}

Intermittency in turbulence is considered to be an outcome of the dynamical cascade of the turbulent fluctuations. So far, we have described analytically a local representation of the turbulence field. We now try to derive a model equation of cascade of the fluctuation given above. Substituting (6) to $\omega$ in (3) and using (4) and (5), we obtain

$$
-g(\xi) \dot{\lambda}=p(\xi) \lambda+G(\xi) \lambda^{h}+\nu f^{\prime \prime}(\xi) \frac{1}{\lambda}
$$

by dropping the common factor $s_{0} \lambda_{0}^{\gamma} \lambda^{-\gamma-1}$, where

$$
\begin{aligned}
& g(\xi)=\gamma f(\xi)+\left(\xi+\xi_{0}\right) f^{\prime}(\xi), \quad G(\xi)=\frac{d}{d \xi}\left[\Psi_{z}\left(\frac{x}{\lambda}, \frac{z}{\lambda}\right) f(\xi)\right] \\
& p(\xi)=C f(\xi)-A\left(\xi+\xi_{0}\right) f^{\prime}(\xi)
\end{aligned}
$$

where $\Psi_{z} \approx a x^{h}$. Let us now introduce the normalized variable

$$
\kappa(t)=\lambda(0) / \lambda(t)
$$


signifying a typical wave number of the test field with unit initial value, $\kappa(0)=1$. The equation for $\kappa(t)$ is readily obtained from (13) by multiplying $\lambda_{0} / \lambda^{2}$ as

$$
\frac{d}{d t} \kappa=\frac{p(\xi)}{g(\xi)} \kappa+\lambda_{0}^{h-1} \frac{G(\xi)}{g(\xi)} \kappa^{2-h}+\nu \frac{1}{\lambda_{0}^{2}} \frac{f^{\prime \prime}(\xi)}{g(\xi)} \kappa^{3}
$$

From now we examine only the general feature of the test field, i.e. the scaling parameter $\kappa(t)$. So that we approximate the function $f(\xi)$ by $f(0) \exp \left(-\frac{1}{2} \xi^{2} / b^{2}\right)$ and restrict our consideration to the behavior at $\xi=0$ (and $z=0$ ). Since $f^{\prime \prime}(0)=$ $-b^{-2} f(0)$, the parameter $b^{-2}$ signifies the magnitude of $f^{\prime \prime}(\xi)$. In addition, we have $f^{\prime}(0)=0, g(0)=\gamma f(0), G(0) \approx a h \xi_{0}^{h-1} f(0), p(0)=C f(0)$. Thus we obtain from (15)

$$
\frac{d}{d \tau} \kappa(\tau)=C \kappa+L_{0} \kappa^{2-h}-\nu k_{0}^{2} \kappa^{3}
$$

where $\tau=t / \gamma, L_{0}=\Psi_{x z}\left(x_{0}, 0\right) \approx a h x_{0}^{h-1}=a h\left(\lambda_{0} \xi_{0}\right)^{h-1}$ and $k_{0}=1 / b \lambda_{0}$. Since $-0.5<h<1.0$, the exponent of the second term is $1<2-h<2.5$, falling in between the first and third terms.

It is interesting to find that the form of the equation (16) for $\kappa$ is equivalent to that of She (1991) except for the coefficients. Especially noted that the same exponent $2-h$ appears at the second term. Kraichnan's model equation takes the form , $\dot{J}=\left|s_{0}\right| J^{2}-\nu k_{d}^{2} J^{3}$, where $J\left(s_{0}, t\right)=s(t) / s_{0}$ for a transverse shear $s$, and $k_{d}$ is a characteristic dissipation wave number for $s_{0}$. One of the major differences lies in that the fractional exponent $h$ is not included in this model, instead a quadratic term is taken into account to express the nonlinear straining. In the present analysis (and She's model, too), the fractional exponent is adopted to represent the fact that the turbulent straining has a singular nature not expressible by an integer power.

The idea of Kraichnan (1990) is as follows. The field variables like the velocity gradients develop under the nonlinear dynamical equation representing the cascade and viscous damping, during which the probability measure of the distribution is kept unchanged, probably due to the rapid process of the cascade. So that the distribution is distorted when expressed in terms of the dynamically evolving variable, due to a nonlinear dependence on the initial value. This is a dynamical mapping of the distribution function (the mapping closure, due to Kraichnan). Let $s$ denote a field variable, and suppose that $s$ has an initial variable $s_{0}$ with the initial distribution given by the gaussian function, $P_{0}\left(s_{0}\right) \sim \exp \left[-s_{0}^{2}\right]$. The probability distribution function will be given by

$$
P(s)=P_{0}\left(s_{0}\right) \frac{\partial s_{0}}{\partial s}
$$

Kraichnan represented as $s=s_{0} J\left(s_{0}, t\right)$ and sought the solution $J\left(s_{0}, t\right)$ by his nonlinear equation. 


\section{An improvement of the model dynamics}

\subsection{Improved representation}

In the previous form of the test field (5) which has only the $y$ component of velocity $v^{(t)}=F_{x}(x, t)$, the longitudinal velocity derivative $\partial v^{(t)} / \partial y$ vanishes identically. In order to examine more general properties of the cascade including the longitudinal derivative as well as self-interaction, we consider the test field in the form,

$$
\psi(x, y, t)=y F(x, t)
$$

Then the vorticity is given by

$$
\omega=y F_{x x}(x, t), \quad=s_{0} \frac{y}{\lambda_{0}}\left(\frac{\lambda_{0}}{\lambda(t)}\right)^{\gamma} f(\xi),
$$

where the function $F_{x x}(x, t)$ is assumed to be of the same form as $(6)$ before. The velocity fields in this case take the form,

$$
u=A x-\Psi_{z}-F(x, t), \quad v=B y+y F_{x}(x, t), \quad u=C z+\Psi_{x}
$$

Therefore the longitudinal derivatives are

$$
\frac{\partial u}{\partial x}=A-\Psi_{x z}-F_{x}, \quad \frac{\partial v}{\partial y}=B+F_{x}, \quad \frac{\partial w}{\partial z}=C+\Psi_{x z}
$$

and the lateral derivatives are

$$
\frac{\partial u}{\partial z}=-\Psi_{z z} \sim-x^{h-2} z, \quad \frac{\partial v}{\partial x}=y F_{x x}=\omega, \quad \frac{\partial w}{\partial x}==\Psi_{x x} \sim x^{h-2} z
$$

Substituting (18) in (3) and using (17), we obtain

$$
-g(\xi) \dot{\lambda}=p(\xi) \lambda+\dot{G}(\xi) \lambda^{h}+q(\xi) \lambda^{-\gamma+2}+\nu f^{\prime \prime}(\xi) \frac{1}{\lambda}
$$

where the third term $q \lambda^{-\gamma+2}$ newly appears to represent the self-interaction, and

$$
\begin{aligned}
& p(\xi)=(C-B) f(\xi)-A\left(\xi+\xi_{0}\right) f^{\prime}(\xi) \\
& q(\xi)=s_{0}\left[f^{\prime}(\xi) \phi(\xi)-f(\xi) \phi^{\prime}(\xi)\right]
\end{aligned}
$$

with the definition $\phi^{\prime \prime} \equiv f(\xi)$. The coefficients $g(\xi)$ and $G(\xi)$ are the same as before.

The equation of $\kappa(\tau)=\lambda(0) / \lambda(\tau)$ corresponding to (16) is now obtained as

$$
\frac{d}{d \tau} \kappa(\tau)=(C-B) \kappa+L_{0} \kappa^{2-h}+q_{0} \kappa^{\gamma}-\nu k_{0}^{2} \kappa^{3}
$$


where $q_{0}=s_{0} f(0)=s_{0}$, by taking as $f(0)=1$, and $\gamma$ is a free parameter unspecified so far. The coefficient $q_{0}=s_{0}$ denotes the initial magnitude of the test field vorticity, that is the transverse shear.

\subsection{Remarks}

(a) Scaling exponent $\gamma$

The equations for the vorticity $\omega$ and its derivative $\omega_{x}$ are

$$
\dot{\omega}=\left(C+\Psi_{x z}\right) \omega, \quad \dot{\omega}_{x}=2\left(C+\Psi_{x z}\right) \omega_{x}+B \omega_{x}+\Psi_{x x z} \omega
$$

from (3) for the inviscid motion $(\nu=0)$, where the over-dot denotes the convective material derivative. Hence we have

$$
-2 \frac{\dot{\omega}}{\omega}+\frac{\dot{\omega}_{x}}{\omega_{x}}=B+\Psi_{x x z} \frac{\omega}{\omega_{x}}
$$

Assuming that $\omega \sim \lambda^{-\gamma}(t), \omega_{x} \sim \lambda^{-\gamma-1}(t)$ and $\Psi_{x x z} \sim a h(h-1)\left(\lambda \xi_{0}\right)^{h-2}$ and neglecting $B$, we obtain

$$
(\gamma-1) \frac{\dot{\lambda}}{\lambda} \approx a h(h-1)\left(\lambda \xi_{0}\right)^{h-2} \lambda
$$

The equation (12) implies $\dot{\lambda} / \lambda \sim-a h\left(\lambda \xi_{0}\right)^{h-1}$ (neglecting $A$ ). Hence we have $\gamma \approx$ $1+c(1-h)$ where $c=1 / \xi_{0}>0$. Since $h<1, \gamma>1$. It is likely that $2-h \geq \gamma(>1)$, since the vortex stretching by the singular component will be more effective than the self-stretching for the cascade. Here we take $c=1$, therefore

$$
\gamma=2-h
$$

This means that the vorticity (18) has the same behavior as the lateral derivative of $\mathbf{v}^{(s)}$ varying like $x^{h-2}$ as $x$ decreases (see (21)). For the value of (27), the second and third terms on the right hand side of (24) are put together with the common factor $\kappa^{2-h}$ and the coefficient given by $\left(L_{0}+s_{0}\right)$ which is taken to be positive. The two terms are considered to be of the same order : $L_{0} \sim s_{0}$.

(b) Skewness

The cubic sum of the longitudinal derivatives is

$$
\begin{aligned}
\Sigma_{3} & =\left(A-\Psi_{x z}-F_{x}\right)^{3}+\left(B+F_{x}\right)^{3}+\left(C+\Psi_{x z}\right)^{3} \\
& =S_{3}+3\left(-\left(A-\Psi_{x z}\right)^{2}+B^{2}\right) F_{x}-3\left(C+\Psi_{x z}\right) F_{x}^{2}
\end{aligned}
$$

where $S_{3}$ is given by (11). In the case $C>0$ and $F_{x}>0$ (given above), the third term on the right hand side is negative, while the second term becomes negative for $A \leq B<0$. In particular it is expected that at the peak position of the test field, 
the new negative contributions exceed in magnitude the positive $S_{3}$, although each of the three terms of (20) takes positive or negative values. Thus we find that our field is simulating qualitatively the property $(b)$ described in the introduction for the probability density function. Over most points (in the neighborhood of the origin and $|z|<x)$ the cubic sum will be given approximately by $S_{3}$ which is positive, but not very large. However at points in a region near the peak of the test field which could be intensified by the cascade, the cubic sum $\Sigma_{3}$ becomes negative. This infers qualitatively the relation of the skewness factor and the distribution function mentioned in $\S 1$, by supposing that the time average of the longitudinal derivative at a fixed point in the experiment corresponds to the spatial average in the present model.

\section{Distribution functions of velocity derivatives}

\subsection{Near-exponential distributions of lateral and longitudinal derivatives}

(a) Growth of the scale parameter $\kappa$

As far as the condition for the initial growth $\dot{\kappa}(0)>0$ is satisfied, $\kappa(t)$ tends to a non-zero stationary value $\kappa_{*}=\kappa(\infty)$ at which the right hand side of (24) vanishes. For large values of $L_{0}+s_{0}$, the scale parameter $\kappa(t)$ will grow sufficiently so that the stationary value $\kappa_{*}$ is given by the balance of the last three terms of (24), that is we have $\left[\left(L_{0}+s_{0}\right) \kappa_{*}^{2-h}-\nu k_{0}^{2} \kappa_{*}^{3}\right] \approx 0$. Thus we obtain

$$
\kappa_{*} \approx\left(\frac{L_{0}+s_{0}}{\nu k_{0}^{2}}\right)^{1 /(1+h)}
$$

\section{(b) Lateral derivative}

As shown in (21), the lateral shear $s(t)$ is given by $\omega=y F_{x x}$. Therefore from the expression (18) we find the scaling relation (for $\xi=0$ ):

$$
\frac{s(t)}{s_{0}} \approx \kappa^{2-h}(t)
$$

for $y$ of the order of $\lambda_{0}$, using (27). For the stationary state, we obtain

$$
\frac{s_{*}}{s_{0}} \approx \kappa_{*}^{2-h} \sim s_{0}^{(2-h) /(1+h)},
$$

where $s_{*}=s(\infty)$, assuming $L_{0} \sim s_{0}$ in (36) (except for the proportionality constant). Hence

$$
s_{0} \sim s_{*}^{\alpha(h) / 2}, \quad \text { with } \quad \alpha(h)=\frac{2}{3}(1+h) .
$$


Thus the distribution of $s_{*}$ will be

$$
P\left(s_{*}\right)=P_{0}\left(s_{0}\right) \frac{\partial s_{0}}{\partial s_{*}} \sim s_{*}^{\mu_{\alpha}} \exp \left[-s_{*}^{\alpha}\right]
$$

with $\mu_{\alpha}=-(2-h) / 3$. For $-\frac{1}{2}<h<1$, we obtain $\frac{1}{3}<\alpha(h)<\frac{4}{3}$. The Kolmogorov scaling $h=1 / 3$ gives $\alpha=8 / 9$. This leads to a slower-than exponential (i.e. $\alpha<$ 1) distribution with respect to $s_{*}$ and predicts an upward flare of the skirt of the distribution observed in the linear-log plot from the simulations of turbulence (She et al. 1988; Hosokawa \& Yamamoto 1989; Kraichnan 1990; Vincent \& Meneguzzi 1991).

\section{(c) Longitudinal derivative.}

The longitudinal derivative $g$ concerned with the cascade is given by $\partial u / \partial x$ or $\partial v / \partial y$ of $(20)$, which include $F_{x}$. The component of intermittency $\sigma(t)$ is

$$
\sigma(t)=F_{x}=s_{0}\left(\frac{\lambda_{0}}{\lambda(t)}\right)^{1-h} \phi^{\prime}(\xi), \quad \phi^{\prime}(\xi)=\int_{-\infty}^{\xi} f\left(\xi^{\prime}\right) d \xi^{\prime}
$$

from (27). Note that $\partial u / \partial x$ includes $-F_{x}$, while $\partial v / \partial y$ includes $+F_{x}$. An argument similar to that for the lateral derivative suggests the relation

$$
\frac{\sigma_{*}}{\sigma_{0}} \approx \kappa_{*}^{1-h} \sim\left(\frac{L_{0}+s_{0}}{\nu}\right)^{(1-h) /(1+h)}
$$

where $\sigma_{0}=s_{0} \phi^{\prime}(0)>0$ and $\sigma_{*}=\sigma(\infty)$. Note that, for a large $\sigma_{0}, \partial v / \partial y$ will be positive, if $\partial u / \partial x$ is negative.

For the derivative $g=\partial u / \partial x$, the initial value will be negative with the value $g_{0}=A-\Psi_{x z}\left(x_{0}, 0\right)-\sigma_{0} \approx-\sigma_{0}\left(1+c_{-}\right)$where $c_{-}=\left|A-\Psi_{x z}\right| / \sigma_{0}$. The final value will be

$$
g_{*} \approx-\sigma_{*} \approx-\sigma_{0} \dot{\kappa}_{*}^{1-h}
$$

from (33). Since $L_{0}=\Psi_{x z}\left(x_{0}, 0\right)$, we have $g_{0} \approx-L_{0}-s_{0} \phi^{\prime}(0)+A \approx-L_{0}-s_{0}$, assuming $\phi^{\prime}(0)=O(1)$ and neglecting $A$. The equation (28) suggests $\kappa_{*} \sim\left|g_{0}\right|^{1 /(1+h)}$. Thus we have

$$
\left|g_{*}\right| \sim \sigma_{0}\left|g_{0}\right|^{e(h)} \approx\left(1+c_{-}\right)^{e(h)} \sigma_{0}^{2 / \beta(h)}
$$

from (42), where

$$
\beta(h)=1+h, \quad \varepsilon(h)=\frac{1-h}{1+h} .
$$

This corresponds to the negative side of the $g_{*}$ distribution. On the other hand, the initial value of $g=\partial v / \partial y$ will be positive with $g_{0}=B+\sigma_{0} \approx \sigma_{0}\left(1-c_{+}\right)>0$ for a 
large fluctuation $\sigma_{0}$, where $c_{+}=|B| / \sigma_{0}$. It is assumed that $1>c_{+}>0$ and $c_{+}<c_{-}$. The final value will be

$$
g_{*} \approx+\sigma_{*} \sim \sigma_{0} g_{0}^{e(h)} \approx\left(1-c_{+}\right)^{e(h)} \sigma_{0}^{2 / \beta(h)}
$$

corresponding to the positive side of $\boldsymbol{g}_{*}$.

Suppose that $\sigma_{0}$ is a gaussian random variable with the probability $Q_{0}\left(\sigma_{0}\right) \sim$ $\exp \left[-\sigma_{0}^{2}\right]$. Then the distribution of $g_{*}$ will be

$$
Q\left(g_{*}\right)=Q_{0}\left(\sigma_{0}\right) \frac{\partial \sigma_{0}}{\partial g_{*}} \sim \begin{cases}g_{*}^{\mu_{\beta}} \exp \left[-k_{-} g_{*}^{\beta}\right] & \left(g_{*}<0\right) \\ g_{*}^{\mu_{\beta}} \exp \left[-k_{+} g_{*}^{\beta}\right] & \left(g_{*}>0\right)\end{cases}
$$

where $\mu_{\beta}=-(1-h) / 2$,

$$
k_{-}=\left(\frac{1}{1+c_{-}}\right)^{1-h}, \quad k_{+}=\left(\frac{1}{1-c_{+}}\right)^{1-h},
$$

$k_{-}$being smaller than $k_{+}$. This kind of asymmetry is observed in both experiments (e.g. Gagne 1990) and numerical simulations (e.g. Hosokawa \& Yamamoto 1989; Yamamoto \& Kambe 1991). For $-\frac{1}{2}<h<1$, we obtain $\frac{1}{2}<\beta(h)<2$. In the Kolmogorov scaling $h=1 / 3$, we have $\beta=4 / 3$. This leads to a steeper-than exponential (i.e. $\beta>1$ ) distribution with respect to the longitudinal derivative $\sigma_{*}$. This behavior too is observed in the numerical simulations as well as in the experiment (Makita 1991).

\subsection{Flatness factors}

Once the probability distribution functions (pdfs, in short) are found by the method described above, it is possible to estimate the flatness factors of the velocity derivatives. Now we consider the $n$-th order longitudinal derivative $g_{n}(t) \equiv$ $g(n, t)=\partial^{n} u / \partial x^{n}$. The (normalized) pdf is

$$
Q_{0}\left(\sigma_{0}\right) d \sigma_{0}=\frac{1}{\sqrt{\pi}} e^{-\sigma_{*}^{\beta}} \frac{\beta}{2} \sigma_{*}^{\frac{1}{2} \beta-1} d \sigma_{*} \rightarrow P(x) d x
$$

where

$$
\begin{gathered}
P(x)=\frac{1}{\sqrt{\pi}} x^{-\frac{1}{2}} e^{-x}, \quad x=\left|\sigma_{*}\right|^{\beta} \\
\beta=\beta(n, h)=\frac{2(1+h)}{\delta(n)+1+h}, \quad \delta(n)=n-h,
\end{gathered}
$$


( $x$ takes only positive values, while $g_{*}$ takes either of positive or negative values). Setting $g_{*}(n)=k_{n} \sigma_{*}(n)$, we obtain

$$
\begin{aligned}
& <g_{*}(n)^{2}>=k_{n}^{2} \int_{0}^{\infty} x^{\frac{2}{\beta}} P(x) d x=\frac{k_{n}^{2}}{\sqrt{\pi}} \Gamma\left(\frac{3}{2}+\frac{\delta(n)}{1+h}\right), \\
& <g_{*}(n)^{4}>=k_{n}^{4} \int_{0}^{\infty} x^{\frac{4}{\beta}} P(x) d x=\frac{k_{n}^{4}}{\sqrt{\pi}} \Gamma\left(\frac{5}{2}+\frac{2 \delta(n)}{1+h}\right),
\end{aligned}
$$

where (40) and (41) are used, and $\Gamma(x)$ is the gamma function. Thus the flatness factor is given by

$$
F_{n}(h)=\frac{<g_{n}^{4}>}{<g_{n}^{2}>^{2}}=\sqrt{\pi} \frac{\Gamma\left(\frac{5}{2}+2 D(n, h)\right)}{\left[\Gamma\left(\frac{3}{2}+D(n, h)\right)\right]^{2}}, \quad D(n, h)=\frac{\delta(n)}{1+h} .
$$

It is readily shown that, if $D(n, h)=0, F_{n}$ takes the gaussian value 3 . Hence the function $D(n, h)=\delta(n) /(1+h)$ characterizes the degree of non-gaussian statistics. In the above case, we have

$$
\delta(n)=n-h, \quad D(n, h)=\frac{n-h}{1+h} .
$$

For $h=1$, we obtain $F_{1}(1)=3$. The value $h=1 / 3$ of the Kolmogorov scaling leads to $D\left(n, \frac{1}{3}\right)=(3 n-1) / 4$. Therefore

$$
F_{1}\left(\frac{1}{3}\right)=\sqrt{\pi} \frac{\Gamma\left(\frac{7}{2}\right)}{[\Gamma(2)]^{2}} \approx 5.9, \quad F_{2}\left(\frac{1}{3}\right)=\sqrt{\pi} \frac{\Gamma(5)}{\left[\Gamma\left(\frac{11}{4}\right)\right]^{2}} \approx 16.4, \quad F_{3}\left(\frac{1}{3}\right)=\sqrt{\pi} \frac{\Gamma\left(\frac{13}{2}\right)}{\left[\Gamma\left(\frac{7}{2}\right)\right]^{2}} \approx 46.2 .
$$

These values appear to be high, compared with the measurements quoted in $(a)$ of the introduction. However a numerical simulation yielded $F_{1}=7.55, F_{2}=14.4$ and $F_{3}=16.1$ (Yamamoto \& Kambe 1991), the first two being not far from the present results.

\section{Summary and discussion}

A dynamical model of intermittency in turbulence is presented in which the velocity field is represented locally in physical space and consists of three components: a regular field, a singular field with a fractional scaling exponent $h$ and a test field. The first two are supposed to represent large-scale inertial range velocity field and the last one an intermittency which is a cascading component. Nonlinear ordinary differential equations are derived to describe growth of the inverse scale $\kappa$ of the test field. Final equilibrium value of $\kappa$ depends on the initial value $s_{0}$ of a velocity derivative, the fractional exponent $h$ and the viscosity. In a particular case, the equation reduces 
to that of She (1991). The present analysis is based on an analytical representation of the velocity field. This suggests that the lateral derivatives of the velocity of the intermittency component scales like $\kappa^{2-h}$, while the scaling (dynamical) exponent of She is $1-h$ (according to his notation, scaling like $J^{1-\alpha}$ ).

In the present formulation the longitudinal derivative $\sigma$ of the cascading test field is characterized by a scaling exponent smaller by one than that of the lateral derivatives. Therefore the variable $\sigma$ scales like $\kappa^{1-h}$ and the corresponding pdf is given by $\exp \left(-|\sigma|^{\beta}\right)$ with $\beta=1+h$. In the Kolmogorov scaling we have $\beta=4 / 3$ which corresponds to a steeper-than exponential pdf. The present model can predict the asymmetry of the distribution function of the longitudinal derivative, which is consistent with the observed statistics.

From the analytical expression of the intermittency component, we have an estimate of the $n$-th order derivative. The flatness factor obtained from the pdf for the $n$-th order derivative increases with the order $n$. The estimated values (for $n=1$ and 2) coincide with the corresponding ones of numerical simulation, but the observed values are smaller than the present one.

One of the points of the present study is that the velocity field includes a component which has a fractional scaling exponent, giving a singular behavior. This is based on the views that the fractional scaling behavior is predicted by the argument given at the end of $\S 1$, further that straining of the test field only by the regular component (Kambe 1983, 1984) is insufficient to describe the cascade in turbulence. The singular component $\mathrm{v}^{(s)}$ gives rise to a locally intensified rate of vortex stretching that could result in the intermittency. This formulation may be considered to be a realization in an analytical form of the idea of She (1991 a).

At first sight, it may appear that the present representation of the velocity is given a particular form. However the following arguments indicate that the velocity has a fairly general character more than its appearance. The regular field $\mathbf{v}^{(r)}$ has a general local expression. The direction of stretching of the field $\mathrm{v}^{(s)}$ is taken in the $z$ direction that does not hurt its generality, but it is assumed to have a two-dimensional form that is not always the case. Another restriction is that the vorticity of the test field coincides with the $z$ axis. In turbulent field there is of course substantial probability of such an arrangement. Once the assumed field is realized, then the cascade mechanism becomes active and a large amplitude fluctuation appears with a fair probability. In unfavorable arrangements the fluctuations will stay at small amplitudes with near-gaussian probability. 


\section{References}

Bacry, E., Arneodo A., Frisch U., Gagne, Y. \& Hopfinger, E. (1990) Wavelet analysis of fully turbulent data and measurement of scaling exponents, in Turbulence and coherent structures (eds. Métais \& Lesieur, Kluwer acad. publ.).

Batchelor, G.K. (1953) The Theory of Homogeneous Turbulence, chap. viii, (Cambridge University Press, London).

Batchelor, G. K. \& Townsend, A. A. (1947) Proc. R. Soc. A 190, 534-550.

Frisch, U. \& Parisi, G. (1985) in Turbulence and Predictability in Geophysical Fluid Dynamics and Climate Dynamics (eds. Ghil, Benzi \& Parisi, North-Holland) 84-88.

Gagne, Y. (1990) Properties of fine scales in high Reynolds number turbulence, in Advances in Turbulence 3 (European Turbulence Symposium at Stockholm).

Hosokawa, I. \& Yamamoto, K. (1989) J. Phys. Soc. Jpn. 58, 20-23.

Kambe, T. (1983) J. Phys. Soc. Jpn. 52, 834-841.

Kambe, T. (1984) J. Phys. Soc. Jpn. 53, 13-15.

Kambe, T. (1986) Fluid Dyn. Res. 1, 21-31.

Kida, S. \& Murakami, Y. (1989) Fluid Dyn. Res. 4, 347-370.

Kraichnan, R. H. (1990) Phys. Rev. Lett. 65, 575-578.

Makita, H. (1991) Fluid Dyn. Res. 8 .

Monin, A.S. \& Yaglom, A.M. (1975) Statistical Fluid Mechanics vol. 2 (MIT Press). Proudman, I. \& Reid, W.H. (1954) Phil. Trans. Roy. Soc. A 247, 163-189.

Rose, H.A. \& Sulem, P.L. (1978) Journal de Physique 39, 441-484.

She, Z.-S. (1991 a) Phys. Rev. Lett. 66, 600-603.

She, Z.-S. (1991 b) Fluid Dyn. Res. 8 .

She, Z.-S., Jackson, E. \& Orszag, S.A. (1988) J. Sci. Comput. 3, 407-434.

She, Z.-S. \& Orszag, S.A. (1991) Phys. Rev. Lett. 66, 1701-1704.

Sheih, C.M., Tennekes, H. \& Lumley, J.L. (1971) Phys. Fluids 14, 201-2215.

Van Atta, C.W. \& Chen, W.Y. (1970) J. Fluid Mech. 44, 145-159.

Vincent, A. \& Meneguzzi, M. (1991) J. Fluid Mech. 225, 1-20.

Yamamoto, K. \& Hosokawa, I. (1988) J. Phys. Soc. Jpn. 57, 1532-1535.

Yamamoto, K. \& Kambe, T. (1991) Fluid Dyn. Res. 8 . 